\title{
A comparison of serial computed tomography and functional change in bronchiectasis
}

\author{
R.E. Sheehan*, A.U. Wells", S.J. Copley*, S.R. Desai*, S.J. Howling*, P.J. Cole ${ }^{\star}$, R. Wilson", \\ D.M. Hansell*
}

A comparison of serial computed tomography and functional change in bronchiectasis. R.E. Sheehan, A.U. Wells, S.J. Copley, S.R. Desai, S.J. Howling, P.J. Cole, R. Wilson, D. M. Hansell. C) ERS Journals Ltd 2002.

ABSTRACT: In bronchiectasis the morphological determinants of (marginal) fluctuations in pulmonary function tests are uncertain. The aim of the present study was to evaluate serial computed tomography (CT) changes in relation to pulmonary function trends in patients with bronchiectasis.

The relationships between pulmonary function indices and CT scans in 48 adult patients with bronchiectasis were evaluated at baseline and at follow-up, at a median interval of 28 months (range 6-74 months). Two independent observers semiquantitatively scored CT features of bronchial and small airways disease.

At initial assessment, the severity of airflow obstruction was linked primarily to the extent of mosaic attenuation. However, serial changes in pulmonary function indices were only associated with serial changes in mucous plugging scores. Alterations in mucous plugging on serial CT were associated with changes in the severity of bronchiectasis and bronchial wall thickness. Greater severity of all three morphological abnormalities at baseline CT were predictive of significant declines in forced expiratory volume in one second, with severe bronchial wall thickness being the most adverse prognostic determinant.

Variations in mucous plugging on computed tomography correlate with minor fluctuations in pulmonary function tests in bronchiectasis. However, the severity of bronchial wall thickness is the primary determinant of subsequent major functional decline.

Eur Respir J 2002; 20: 581-587.

\author{
*Dept of Radiology, ${ }^{\text {*Interstitial Lung }}$ \\ Disease Unit and "Host Defence Unit, \\ Royal Brompton Hospital, London, \\ UK. \\ Correspondence: D.M. Hansell \\ Dept of Radiology \\ Royal Brompton Hospital \\ Sydney Street \\ London \\ SW3 6NP \\ UK \\ Fax: 442073518098 \\ E-mail: d.hansell@rbh.nthames.nhs.uk
}

Keywords: Bronchiectasis

computed tomography

pulmonary function

Received: October 42001

Accepted after revision: March 62002
Bronchiectasis is a variably progressive disease that is usually monitored clinically using reported symptoms and pulmonary function tests (PFTs). However, pulmonary function indices are insensitive in the detection of regional abnormalities [1]. Moreover, fluctuations in pulmonary function may reflect variations in a number of different morphological abnormalities, including the severity of bronchial dilatation, the degree of bronchial wall thickness, the severity of small airways involvement and the volume of retained secretions in small and large airways [2-6]. Major changes in pulmonary function indices may be required before significant irreversible structural damage can be identified confidently. Thus, a sensitive morphological tool is needed in order to evaluate the significance of lesser fluctuations in pulmonary function.

High resolution computed tomography (CT) has an established role in the detection of bronchiectasis [7-9]. A number of individual morphological features identifiable on CT in patients with bronchiectasis have functional significance [10-12]. Thus, there are potential roles for serial CT in mapping the evolution of bronchiectasis and providing explanations for fluctuations in PFTs during follow-up. To date, there have been no longitudinal studies on bronchiectasis that have evaluated the relationship between variations in pulmonary function indices and changes on $\mathrm{CT}$, except in cystic fibrosis $[13,14]$. The aim of the present study was to evaluate serial morphological changes in relation to pulmonary function trends in patients with bronchiectasis.

\section{Patients and methods}

\section{Patients}

The study population consisted of patients referred to the current authors' institution with chronic sputum production, who had two high-resolution computed tomography (HRCT) scans and formal PFTs between January 1991 and September 1999. The time interval between CT scans was 6-74 months (median 28 months). Patients who did not have PFTs within 2 months of the HRCT were excluded. The final study group consisted of 48 patients (idiopathic bronchiectasis $\mathrm{n}=30$, primary ciliary dyskinesia $\mathrm{n}=8$, hypogammaglobulinaemia $n=5$, allergic bronchopulmonary aspergillosis $n=4$, rheumatoid arthritis $n=1$ ). None of the patients had cystic fibrosis (excluded by a normal 
sweat test) or a clinical history of asthma. There

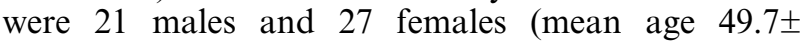
$14.0 \mathrm{yrs})$.

\section{Pulmonary function tests}

Functional indices (forced expiratory volume in one second (FEV1), forced vital capacity (FVC), total lung capacity (TLC), midexpiratory flow at $25 \%$ of the vital capacity (MEF25), carbon dioxide diffusing capacity of the lung $(D \mathrm{~L}, \mathrm{CO})$, carbon monoxide transfer coefficient $(\mathrm{KCO}))$ were expressed as percentages of values predicted for the patient's age, sex and height [15]. Spirometric volumes were measured using a rolling seal spirometer (P.K. Morgan Ltd, Kent, UK). Lung volumes were measured using a constant volume body plethysmograph (Masterlab equipment, E. Jaeger UK Ltd, Market Harborough, UK). Indices of gas transfer were measured using a carbon monoxide single breath technique; gas transfer results were adjusted for haemoglobin (Model B; P.K. Morgan Ltd and 6200 Autobox DL; Sensormedics Inc, Yorba Linda, CA, USA).

\section{CT scans}

CT scans were obtained through use of an electron beam CT scanner (Imatron, Inc., San Francisco, CA, USA). Sections of either $1.5 \mathrm{~mm}$ or $3 \mathrm{~mm}$ thickness were acquired at full inspiration (10 $\mathrm{mm}$ intervals) in the supine position. Consecutive scans were of the same section thickness in individual patients. A high spatial resolution algorithm was used to reconstruct images that were photographed at appropriate window settings (level -550 Hounsfield unit (HU); width 1,500 HU). Two observers scored the initial CT scans by consensus given the good interobserver agreement previously reported for CT features of bronchiectasis [16-18]. For the assessment of serial change, the observers scored images independently to allow interobserver variation to be evaluated.

The observers scored the initial CT for each patient at a lobar level (6 lobes; the lingula was regarded as a separate lobe) using a modified Bhalla system [12, 19]. The presence and extent of bronchiectasis was determined according to established CT criteria [7, 20]. Each lobe was scored for the following: 1) extent of bronchiectasis $(0=$ none, $1=$ one or partial bronchopulmonary segment involved, $2=\geqslant$ two or more bronchopulmonary segments involved, $3=$ generalised cystic bronchiectasis); 2) severity of bronchial dilatation $(0=$ normal, $1=$ less than twice the diameter of adjacent pulmonary artery, $2=$ more than twice diameter of adjacent pulmonary artery); 3) severity of bronchial wall thickening $(0=$ normal, $1=0.5 \times$ diameter of adjacent pulmonary artery, $2=0.5-1.0 \times$ diameter of adjacent pulmonary artery, $3=>1.0 \times$ diameter of adjacent pulmonary artery); 4) presence of mucous plugging in large airways ( $0=$ none, $1=$ present $) ; 5$ ) presence of mucous plugging in small airways $(0=$ none, $1=$ present $)$; and 6$)$ extent of decreased attenuation $(0=$ normal, $1=<50 \%$ of lobar volume, $2=>50 \%$ of lobar volume). The total
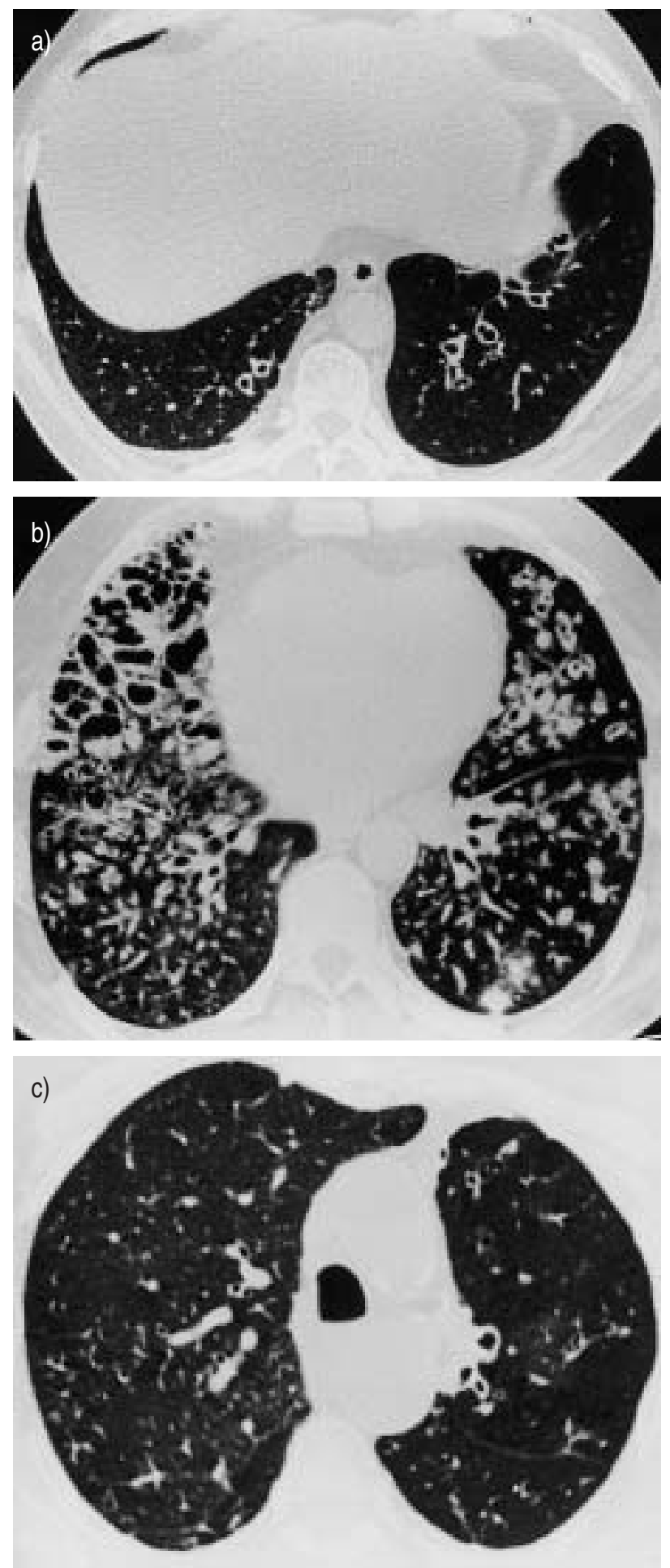

Fig. 1.-Examples of the computed tomography features scored. a) Bronchiectasis in the lower lobes with conspicuous bronchial wall thickening but no mucous plugging. b) Severe cystic bronchiectasis particularly in the right middle lobe with mucous plugging of the large airways (lingula and left lower lobe) and tree-in-bud pattern in the posterobasal segment of the right lower lobe denoting small airways plugging. c) Mild bronchiectasis in the left upper lobe and mosaic attenuation pattern reflecting small airways disease.

lung score for each CT feature was derived by summing the lobar scores. Examples of the features scored are shown in fig. 1 . 
Table 1. - Summary of pulmonary function test data

\begin{tabular}{llc}
\hline Functional index & \multicolumn{1}{c}{ Baseline } & Serial change \\
\hline FEV1 \% pred & $63.3 \pm 24.1$ & $-3.8(-41.6-47.6)$ \\
FVC \% pred & $83.9 \pm 17.1$ & $-1.2(-30.2-49.3)$ \\
FEV $1 /$ FVC & $61.8 \pm 16.9$ & $-0.2(-31.4-25.3)$ \\
$\quad \%$ pred & $78.2 \pm 21.3$ & $-3.8(-46.6-37.7)$ \\
$D \mathrm{~L}, \mathrm{CO} \%$ pred & $99.1 \pm 18.8$ & $-3.8(-23.9-28.6)$ \\
$K \mathrm{CO}$ & $10.0 \pm 1.3$ & $0.1(-1.6-3.6)$ \\
$\mathrm{O}_{2} \mathrm{kPa}$ & $26.3 \pm 4.9-113$ & $-25.8(-68.8-51.3)$ \\
$\mathrm{MEF}_{25}$ &
\end{tabular}

Data are presented as mean \pm SD or median range $\%$. FEV1: forced expiratory volume in one second; FVC: forced vital capacity; $D$ L,CO: carbon monoxide diffusing capacity of the lung; KCO: carbon monoxide transfer coefficient; $\mathrm{PO}_{2}$ : oxygen tension; MEF25: midexpiratory flow at $25 \%$ of vital capacity.

Two observers independently scored the consecutive scans of each patient for longitudinal changes at a bronchopulmonary segmental level. The following variables were evaluated: extent of decreased attenuation, extent of bronchiectasis, amount of bronchial wall thickening, extent of large airway plugging, and extent of small airway plugging. The observers were blinded to the scan dates. A three-point grading system was used (less disease; no change; more disease). Results were agreed upon after each case. The total change for each CT variable was derived by summing the segmental scores. A change of less than two bronchopulmonary segments was recorded as no change.

\section{Data analysis}

Data are expressed as means with SD or medians with ranges, depending upon the normality of distribution. Interobserver variation in serial change in individual features on CT was quantified as the weighted kappa coefficient of agreement ( $\mathrm{Kw})$. Group comparisons were made using Wilcoxon's rank-sum analysis. Univariate correlations between functional indices and CT scores were evaluated using Spearman's rank correlation. The prevalence of serial change in FEV1 and CT was compared using McNemar's Chisquared test (which confines analysis to cases with divergent results). Multivariate analyses were used to identify: 1) initial CT features linked to the initial FEV1 (stepwise regression); and 2) initial CT features associated with a subsequent decline in FEV1 (logistic regression).

\section{Results}

\section{Data at presentation}

Initial pulmonary function indices and changes in pulmonary function indices at follow-up CT are shown in table 1 . The pattern of functional impairment was largely obstructive, with relative preservation of $D \mathrm{~L}, \mathrm{CO}$ and $K \mathrm{CO}$. Average change for the whole cohort was relatively minor at follow-up CT, with the largest declines observed in FEV1 and DL,CO (median change $-3.8 \%$ of baseline for both). However, the range of serial change in individual patients was wide; 12 out of 48 patients $(25 \%)$ had a significant $(>15 \%$ of baseline) decline in FEV1 and four out of 48 patients $(8 \%)$ had a significant improvement.

Initial global CT scores, given as medians (ranges and maximum possible scores), were: extent of bronchiectasis $7(1-16,18)$; bronchial dilatation 5 (1-12, $12)$; bronchial wall thickness $4(0-8,12)$; plugging of large airways $1(0-6,6)$; plugging of small airways 2 $(0-6,6)$; and decreased attenuation $4(0-12,12)$.

As shown in table 2, there were significant relationships between initial CT scores and obstructive pulmonary function indices at presentation. The extent of decreased attenuation correlated more strongly than other CT features with FEV1 (fig. 2) and, on stepwise regression, was the only independent $\mathrm{CT}$ determinant of reduction in FEV1 $\left(\mathrm{p}<0.0005\right.$, equation $\left.\mathrm{R}^{2}=0.38\right)$.

\section{The relationship between change on $C T$ and pulmonary function trends}

Changes in CT features were seen in the majority of patients (table 3). Interobserver agreement for serial CT change was moderate to good (bronchiectasis: $\kappa \mathrm{w}=0.51$; bronchial wall thickness: $\kappa_{\mathrm{w}}=0.55$; decreased attenuation: $\kappa_{w}=0.64$; large airways plugging: $\kappa w=0.66$; small airways plugging: $\kappa_{w}=0.52$ ).

Change in the severity of bronchiectasis was seen more often on CT than in FEV1 (using a 15\% improvement or decline in baseline FEV1 to denote significant change, $\mathrm{n}=12, \mathrm{p}=0.001$ (McNemar's Chi-squared test)). This observation was statistically significant both for improvement $(p<0.01)$ and decline $(p<0.005)$. Changes in the severity of bronchiectasis, bronchial wall

Table 2. - Correlations between initial pulmonary function and initial computed tomography score

\begin{tabular}{llll}
\hline & \multicolumn{1}{c}{ FEV1 $\mathrm{r}$} & FEV1/FVC r & \multicolumn{1}{c}{ MEF25 r } \\
\hline Bronchiectasis & $-0.41(\mathrm{p}<0.005)$ & $-0.38(\mathrm{p}<0.01)$ & $-0.52(\mathrm{p}<0.0005)$ \\
Thickness & $-0.41(\mathrm{p}<0.005)$ & $-0.41(\mathrm{p}<0.005)$ & $-0.46(\mathrm{p}=0.001)$ \\
Large plugs & $-0.40(\mathrm{p}<0.005)$ & $-0.34(\mathrm{p}=0.02)$ & $-0.40(\mathrm{p}<0.01)$ \\
Small plugs & $-0.28(\mathrm{p}=0.05)$ & $-0.21(\mathrm{p}=0.15)$ & $-0.34(\mathrm{p}=0.02)$ \\
Combined plugging score & $-0.36(\mathrm{p}=0.01)$ & $-0.29(\mathrm{p}=0.04)$ & $-0.39(\mathrm{p}<0.01)$ \\
Decreased attenuation (on inspiration) & $-0.55(\mathrm{p}<0.00005)$ & $-0.40(\mathrm{p}<0.005)$ & $-0.56(\mathrm{p}<0.00005)$ \\
\hline
\end{tabular}

FEV1: forced expiratory volume in one second; FVC: forced vital capacity; MEF25: midexpiratory flow at $25 \%$ of vital capacity; r: Spearman's correlation coefficient. 


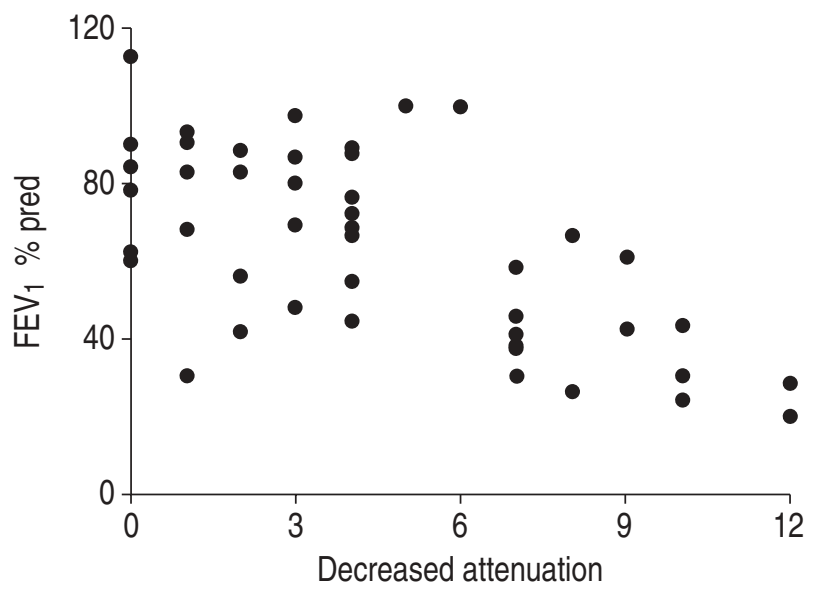

Fig. 2. - Forced expiratory volume in one second $\left(F^{2} V_{1}\right)$ in relation to the extent of decreased attenuation on computed tomography, showing a significant negative correlation $\left(\mathrm{r}_{\mathrm{s}}=-0.55, \mathrm{p}<0.00005\right)$.

Table 3.-Prevalence of progression or regression of individual computed tomography features on serial imaging

Better $\begin{gathered}\text { No } \\ \text { change }\end{gathered}$ Worse Range

\begin{tabular}{lrrrc}
\hline Bronchiectasis & 15 & 6 & 27 & $-11-11$ \\
Decreased attenuation & 5 & 15 & 28 & $-5-18$ \\
Bronchial wall thickness & 19 & 14 & 15 & $-16-6$ \\
Plugging of large airways & 10 & 27 & 11 & $-8-4$ \\
Plugging of small airways & 14 & 21 & 13 & $-13-14$ \\
\hline
\end{tabular}

thickness, decreased attenuation and plugging of small airways were more prevalent than significant change in FEV1 ( $p<0.05$ for all). However, change in FEV1 had a similar prevalence to changes in plugging of large airways.

As shown in table 4, change in the combined plugging score was the serial CT feature most closely associated with change in pulmonary function indices, including FEV1 (fig. 3), FVC and oxygen tension $\left(\mathrm{PO}_{2}\right)$. Changes in large airways plugging were most closely associated with alterations in FEV1 and $\mathrm{PO}_{2}$, whereas changes in small airways plugging were most strongly linked to trends in FVC.

These findings were robust when a comparison was made between 12 patients with a significant decline in

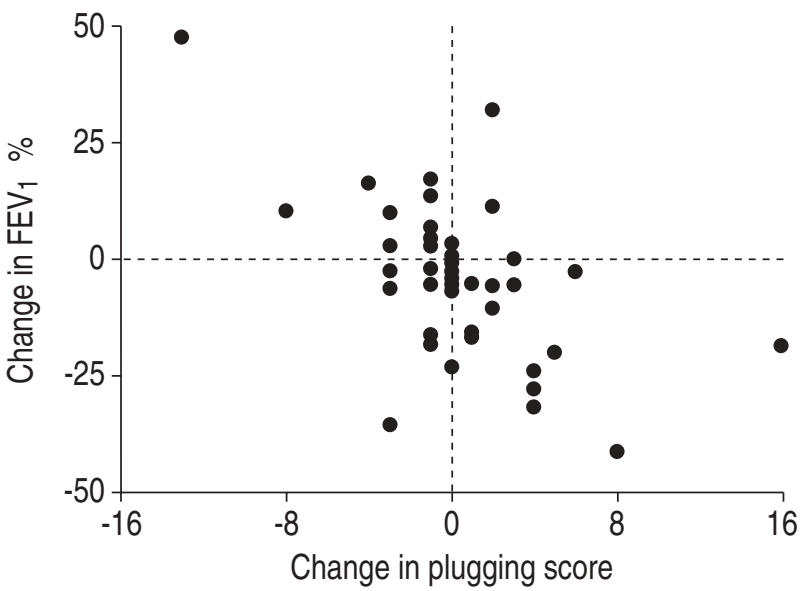

Fig. 3.-Change in airways plugging score in relation to change in forced expiratory volume in one second $\left(F E V_{1}\right)$, showing significant negative correlation $(\mathrm{r}=-0.46, \mathrm{p}<0.001)$.

FEV1 and the remaining 36 subjects. Increases in the combined plugging score were observed more frequently in those who declined (median score 2.5, range -3-16) than in those who remained stable or improved (median score 0, range -13-6) $(\mathrm{p}=0.02)$. However, there were no other significant relationships between changes in CT features and a decline in FEV1.

\section{Correlations between serial CT changes}

Changes in the severity of bronchiectasis were associated with changes in bronchial wall thickness $\left(r_{s}=0.52, p=0.0002\right)$, and both were associated with changes in the combined plugging score $\left(r_{s}=0.45\right.$, $\mathrm{p}=0.001$ and $\mathrm{r}_{\mathrm{s}}=0.44, \mathrm{p}=0.002$, respectively). Changes in the extent of decreased attenuation were not associated with changes in any other CT feature.

The initial CT appearances in relation to subsequent declines in FEVI

Further comparisons were made between 12 patients who had a significant decline in FEV1 and the remaining 36 subjects. Patients who declined had

Table 4. - Relationships between change on serial computed tomography (CT) and pulmonary function trends

\begin{tabular}{llcc}
\hline & FEV1 r & FVC r & $P_{\mathrm{O}_{2} \mathrm{r}}$ \\
\hline Change in: & & & $-0.27(\mathrm{p}=0.18)$ \\
Bronchiectasis & $-0.37(\mathrm{p}=0.01)$ & $-0.32(\mathrm{p}=0.03)$ & $\mathrm{NS}$ \\
Bronchial wall thickness & $-0.25(\mathrm{p}=0.09)$ & $-0.35(\mathrm{p}=0.02)$ & $-0.39(\mathrm{p}<0.05)$ \\
Plugging of large airways & $-0.40(\mathrm{p}<0.005)$ & $-0.34(\mathrm{p}=0.02)$ & $\mathrm{NS}$ \\
Plugging of small airways & $-0.23(\mathrm{p}=0.12)$ & $-0.40(\mathrm{p}<0.005)$ & $-0.42(\mathrm{p}=0.03)$ \\
Combined plugging score & $-0.46(\mathrm{p}<0.001)$ & $-0.52(\mathrm{p}<0.0005)$ & $\mathrm{NS}$ \\
Decreased attenuation & $\mathrm{NS}$ & $\mathrm{NS}$ & $\mathrm{N}$ \\
\hline
\end{tabular}

FEV1: forced expiratory volume in one second; FVC: forced vital capacity; $P_{\mathrm{O}_{2}}$ : oxygen tension; NS: nonsignificant. Changes in carbon monoxide diffusing capacity of the lung and carbon monoxide transfer coefficient were not linked to serial change in any CT feature. 
more severe disease at initial CT, with more extensive bronchiectasis $(\mathrm{p}<0.01)$, thicker bronchial walls $(p<0.0005)$ and higher scores for large airways plugging $(\mathrm{p}=0.02)$, small airways plugging $(\mathrm{p}=0.02)$, and combined plugging scores $(\mathrm{p}<0.01)$. On logistic regression, the sole independent $\mathrm{CT}$ determinant of a subsequent decline in FEV1 was the degree of bronchial wall thickness $(\mathrm{p}<0.005)$.

\section{Discussion}

The results of this study confirm that in patients with bronchiectasis, decreased attenuation of the lung parenchyma on $\mathrm{CT}$, reflecting constrictive bronchiolitis at a pathological level [8], correlates strongly with indices of airflow limitation [11, 12, 21]. Other features that influenced airflow obstruction in the present study included the extent of bronchiectasis and the degree of bronchial wall thickening. However, the correlation between mucous plugging of the airways on the initial CT scan and FEV1/FVC was not as strong. In a study that sought to identify the HRCT features that characterised acute exacerbations in patients with cystic fibrosis, SHAH et al. [14] reported a similar lack of correlation between the extent of mucous plugging of the large and small airways and functional parameters. It might be anticipated that HRCT features most closely linked with functional severity might also be strongly associated with subsequent functional change. However, in the present study, it was serial change of the combined plugging score that most closely correlated with changes in functional indices.

Variations in the burden of secretions in the large and small airways were the major independent determinants of serial changes in pulmonary function indices in the present study. As reported previously in patients with cystic fibrosis [14], secretions visualised on CT were a reversible feature in the patients in this study, with an equal prevalence of increased and decreased mucous plugging at follow-up CT. By contrast, airway plugging in patients with cystic fibrosis tends to be relentlessly progressive [13], a finding that is ascribable to the cardinal role played by mucous impaction in the progression of cystic fibrosis. The current findings can be linked to those of Cochrane et al. [4]. In a small cohort of patients with bronchiectasis, clearance of secretions by rigorous postural drainage was associated with significant increases in pulmonary function indices. The study's findings prompted the current authors to combine large and small airway plugging scores in a total plugging score, which strengthened the observed functional-morphological relationship. Furthermore, changes in the total plugging score were independently associated with arterial oxygen tension, presumably due to ventilation/perfusion mismatch.

By comparison with mucous plugging of the airways, bronchial wall thickness and bronchiectasis were not as strongly associated with serial change in PFTs. However, these three features are, in several respects, intimately linked. A previous study has shown that, with the exception of bronchiectasis, the features are potentially reversible in patients with cystic fibrosis [14]. It is possible that there are differences in the potential for progression of bronchiectasis in cystic fibrosis patients by comparison with the current noncystic fibrosis study group (i.e. the current population with relatively longstanding disease were less likely to have inexorably progressive bronchiectasis). Further evidence that the three features of mucous plugging, bronchial wall thickening and bronchiectasis are tightly linked comes from the observation that all three predicted subsequent decline in FEV1 and that serial change in all three occurred in parallel. It has been postulated that chronic mucous plugging may lead to progressive bronchial damage [21]. It has also been suggested that obliteration of the small airways is the major pathogenetic determinant of later large airways damage and the development of frank bronchiectasis $[8,11,22]$. The results of the current study do not allow a firm conclusion to be drawn about the "first hit" site of damage in patients who subsequently develop bronchiectasis. Specifically, given the nature of the study group, it is not possible to speculate about the early natural history of untreated bronchiectasis.

In contrast to other morphological features, mosaic perfusion rarely regressed on CT. Changes in mosaic perfusion were seldom linked to the evolution of other CT morphological features. The progressive irreversible nature of mosaic perfusion has been highlighted in cystic fibrosis [13, 14], although it has been suggested that it might be minimised by early treatment [13]. Lack of reversibility of mosaic perfusion is not unexpected, as this CT feature is known to denote the histological finding of constrictive bronchiolitis [8], which does not respond to treatment in other contexts. The argument that mosaic perfusion in bronchiectasis might represent emphysema [23] is undermined by the lack of any relationship between the extent of mosaic perfusion and impairment of gas transfer [12]. In the present study, alterations in the extent of mosaic perfusion were not linked to variations in $D \mathrm{~L}, \mathrm{CO}$ or KCO levels.

A study by HelBich et al. [13] suggested that CT is better able to demonstrate serial change than pulmonary function testing. In the current study, serial changes, of any feature, were identified more frequently on $\mathrm{CT}$ than by changes in pulmonary function testing (defined as a 15\% change in FEV1). However, it remains difficult to define clinically significant changes in morphology of the airways (or ancillary signs) on CT. The clinical relevance of those features included within the ambit of "serial change" in the present study need to be validated. A particular problem relates to observer variation in identifying serial, as opposed to static, HRCT observations, which have been documented in the present study for the first time. Given the subjective nature of assessing serial change and the difficulty of making judgements from anatomically incomparable CT sections, observer variation was surprisingly low.

One difficulty in interpreting the current findings is the fact that, overall, changes in lung function indices were minor in most patients. This may reflect the use of systematic treatment, including postural drainage, 
inhaled steroid therapy and the early use of antibacterial agents for infective exacerbations. Thus, it can be argued that the treated course, rather than the natural history, of bronchiectasishas been evaluated in the present study. It is also likely that there was some bias towards patients with more progressive disease, as increasing symptoms and declines in pulmonary function indices undoubtedly stimulated the repetition of CT in some cases. It seems likely that variations in the time interval between CT scans will have had parallel effects upon CT appearances and pulmonary function indices, without necessarily influencing the correlations between functional and morphological change.

Radiation burden to patients is a constraint to the application of HRCT for the routine monitoring of patients with bronchiectasis in clinical practice and future longitudinal investigations. Nevertheless, low dose HRCT protocols have been developed, in which the radiation dose may be reduced by $40 \%-50 \%$ [24, 25]. Low dose HRCT techniques do not preclude the precise quantitation of bronchial dimension and mucous plugging, because these are relatively high contrast structures. However, broad area contrast differences, which notably decreased attenuation of the lung parenchyma reflecting small airways disease, may be less readily detectable on low dose CT images [26]. A significant advantage of $\mathrm{CT}$ is its ability to map the heterogeneous distribution of changes in bronchial morphology, which may change in distribution over time $[27,28]$. Such regional information is not available from routine pulmonary function test data. For the purposes of clinical therapeutic trials, CT may be particularly valuable in the evaluation of mucolytic and other therapies, given the current increased understanding of which features are most likely to show serial change.

In conclusion, variation in the severity of mucous plugging on computed tomography is the morphological feature that most closely mirrors minor changes in pulmonary function tests in patients with bronchiectasis. The severity of bronchial wall thickness is the most important predictor of subsequent major functional decline.

\section{References}

1. Bass H, Henderson JAM, Hecksher T, Oriol A, Anthonisen NR. Regional structure and function in bronchiectasis. Am Rev Respir Dis 1968; 97: 598-609.

2. Stockley RA, Hill SL, Morrison HM, Starkie CM. Elastolytic activity of sputum and its relation to purulence and to lung function in patients with bronchiectasis. Thorax 1984; 39: 408-413.

3. Pande JN, Jain BP, Gupta RG, Guleria JS. Pulmonary ventilation and gas exchange in bronchiectasis. Thorax 1971; 26: 727-733.

4. Cochrane GM, Webber BA, Clarke SW. Effects of sputum on pulmonary function. BMJ 1977; 2: 11811183.

5. Ip M, Lauder IJ, Wong WY, Lam WK, So SY. Multivariate analysis of factors affecting pulmonary function in bronchiectasis. Respiration 1993; 60: 4550 .

6. Wong You Cheong JJ, Leahy BC, Taylor PM, Church SE. Airways obstruction and bronchiectasis: correlation with duration of symptoms and extent of bronchiectasis on computed tomography. Clin Radiol 1992; 45: 256-259.

7. Grenier P, Cordeau MP, Beigelman C. Highresolution computed tomography of the airways. $J$ Thorac Imag 1993; 8: 213-229.

8. Kang EY, Miller RR, Müller NL. Bronchiectasis: comparison of preoperative thin-section CT and pathologic findings in resected specimens. Radiology 1995; 195: 649-654.

9. Hansell DM. Bronchiectasis. Radiol Clin North Am 1998; 36: 107-128.

10. Lynch DA, Newell J, Hale V, et al. Correlation of CT findings with clinical evaluations in 261 patients with symptomatic bronchiectasis. AJR 1999; 173: 5358.

11. Hansell DM, Wells AU, Rubens MB, Cole PJ. Bronchiectasis: functional significance of areas of decreased attenuation at expiratory CT. Radiology 1994; 193: 369-374.

12. Roberts HR, Wells AU, Milne DG, et al. Airflow obstruction in bronchiectasis: correlation between computed tomography features and pulmonary function tests. Thorax 2000; 55: 198-204.

13. Helbich TH, Heinz-Peer G, Fleischmann D, et al. Evolution of CT findings in patients with cystic fibrosis. AJR 1999; 173: 81-88.

14. Shah RM, Sexauer W, Ostrum BJ, Fiel SB, Friedman AC. High resolution CT in the acute exacerbation of cystic fibrosis: evaluation of acute findings, reversibility of those findings, and clinical correlation. AJR 1997; 169: 375-380.

15. Quanjer PH. Standardized lung function testing. Eur Respir J 1993; 6: Suppl. 16, 1-100.

16. Diederich S, Jurriaans E, Flower CDR. Interobserver variation in the diagnosis of bronchiectasis on highresolution computed tomography. Eur Radiol 1996; 6: 801-806.

17. Grenier P, Mourey-Gerosa I, Benali K, et al. Abnormalities of the airways and lung parenchyma in asthmatics: CT observations in 50 patients and interand intra-observer variability. Eur Radiol 1996; 6: 199-206.

18. Reiff DB, Wells AU, Carr DH, Cole PJ, Hansell DM. CT findings in bronchiectasis: limited value in distinguishing between idiopathic and specific types. AJR 1995; 2: 261-267.

19. Bhalla M, Turcios N, Aponte V, et al. Cystic fibrosis: scoring system with thin-section CT. Radiology 1991; 179: 783-788.

20. McGuinness G, Naidich DP. Bronchiectasis: CT/ clinical correlations. Semin US CT MRI 1995; 16: $394-419$.

21. Helbich TH, Heinz-Peer G, Eichler I, et al. Cystic fibrosis: CT assessment of lung involvement in children and adults. Radiology 1999; 213: 537-544.

22. Bedrossian CW, Greenberg SD, Singer DB, Hansen JJ, Rosenberg HS. The lung in cystic fibrosis: a quantitative study including prevalence of pathologic findings among different age groups. Hum Pathol 1976; 7: 195-204.

23. Loubeyre P, Paret M, Revel D, Wiesendanger $\mathrm{T}$, Brune J. Thin-section CT detection of emphysema 
associated with bronchiectasis and correlation with pulmonary function tests. Chest 1996; 109: 360365.

24. Ambrosino MM, Genieser NB, Roche KJ, Kaul A, Lawrence RM. Feasibility of high-resolution, lowdose chest $\mathrm{CT}$ in evaluating the pediatric chest. Pediatr Radiol 1994; 24: 6-10.

25. Mayo JR, Hartman TE, Lee KS, Primack SL, Vedal S, Müller NL. CT of the chest: minimal tube current required for good image quality with the least radiation dose. AJR 1995; 3: 603-607.
26. Zwirewich CV, Mayo JR, Müller NL. Low dose high resolution CT of lung parenchyma. Radiology 1991; 180: 413-417.

27. Brown RH, Herold CJ, Hirshman CA, Zerhouni EA, Mitzner W. In vivo measurements of airway reactivity using high-resolution computed tomography. Am Rev Respir Dis 1991; 144: 208-212.

28. Herold CJ, Brown RH, Mitzner W, Links JM, Hirshman CA, Zerhouni EA. Assessment of pulmonary airway reactivity with high resolution CT. Radiology 1991; 181: 369-374. 